\title{
Local and Global Dynamics in Intrinsically Disordered Synuclein
}

\author{
Nasrollah Rezaei-Ghaleh, Giacomo Parigi, Andrea Soranno, Andrea Holla, Stefan Becker, Benjamin \\ Schuler, Claudio Luchinat *, Markus Zweckstetter *
}

\begin{abstract}
Intrinsically disordered proteins experience a diverse spectrum of motions that are difficult to characterize with a single experimental technique. Here we combine high- and low-field nuclear spin relaxation, nanosecond fluorescence correlation spectroscopy (nsFCS) and long molecular dynamics simulations of alpha-synuclein, a paradigmatic IDP involved in Parkinson disease, to obtain a comprehensive picture of its conformational dynamics. The combined analysis shows that fast motions below 2 ns caused by local dihedral angle fluctuations and conformational sampling within and between Ramachandran substates decorrelate most of the backbone $\mathrm{N}-\mathrm{H}$ orientational memory. However, slow motions with correlation times of up to $\sim 13 \mathrm{~ns}$ from segmental dynamics are present throughout the alpha-synuclein chain, in particular in its Cterminal domain, and global chain reconfiguration occurs on a timescale of $\sim 60$ ns. Our study demonstrates that the combination of high- and low-field nuclear spin relaxation together with nsFCS and molecular dynamics simulations is a powerful strategy to determine residue-specific protein dynamics in IDPs at different time and length scales.
\end{abstract}

Intrinsically disordered proteins (IDPs) constitute a large fraction of the eukaryotic proteome and play key roles in many cellular processes ${ }^{[1]}$. The biological function of IDPs is supported by their high mobility ${ }^{[2]}$, which arises because of the flat energy landscape of IDPs compared to folded proteins. As a result, IDPs are best described as heterogeneous ensembles of rapidly interconverting structures ${ }^{[3]}$ and several ensembles have been proposed based on conformationally averaged structural restraints ${ }^{[4]}$. Frequently, disease-related mutations and posttranslational modifications do not seem to significantly alter the structural properties of IDPs, suggesting that their pathological effect might be influenced by changes in protein dynamics. Nevertheless, a comprehensive picture of the dynamics of IDPs is still lacking. One of the major challenges is that dynamics in IDPs occur over a broad range of length- and timescales that are difficult to cover with a single experimental

[*] Dr. Nasrollah Rezaei-Ghaleh \& Prof. Markus Zweckstetter, University Medical Center Göttingen \& German Center for Neurodegenerative Diseases (DZNE) \& MPI for Biophysical Chemistry, Von-Siebold-Str. 3a, 37075 Göttingen, Germany. E-mail: markus.zweckstetter@dzne.de

Prof. Giacomo Parigi \& Prof. Luchinat, Magnetic Resonance Center (CERM) \& Department of Chemistry "Ugo Schiff", University of Florence, via Sacconi 6, 50121 Sesto Fiorentino, Italy E-mail: luchinat@cerm.unifi.it

Prof. Andrea Sorrano, Washington University in St. Louis, St. Louis, MO 63110 \& University of Zurich, 8057 Zurich, Switzerland

Dr. Andrea Holla \& Prof. Benjamin Schuler, University of Zurich, 8057 Zurich, Switzerland

Dr. Stefan Becker, Max Planck Institute for Biophysical Chemistry, Am Fassberg 11, 37077 Göttingen.

Supporting information for this article (details about MD simulation, calculation of ${ }^{1} \mathrm{H} /{ }^{15} \mathrm{~N}$ relaxation rates, NMR, smFRET / nsFCS experiments) is given via a link at the end of the document. technique. Here we combine three experimental techniques high-field ${ }^{15} \mathrm{~N}$ spin relaxation, low-field ${ }^{1} \mathrm{H}$ relaxometry and nanosecond fluorescence correlation spectroscopy (nsFCS) which are sensitive to motions on different length and timescales, with long molecular dynamics (MD) simulations to obtain insight into local and global dynamics from one to hundreds of nanoseconds in the IDP $\alpha$-synuclein (aS) (SI, Fig. S1).

The starting point of our study was the $16-\mu \mathrm{S}$ MD trajectory for aS, which was previously calculated at $300 \mathrm{~K}$ using the Amber12 force field and the TIP4P-D water model with 2.5 fs time steps and saved at 10 ps time intervals ${ }^{[5]}$. Because of its long simulation time, this MD trajectory extensively samples aS's conformational space and dynamics (Fig. 1a). ${ }^{1} \mathrm{H}$ relaxometry (0.1-50 MHz) of aS was previously recorded ${ }^{[6]}$ (Fig. 1b), while nsFCS and single-molecule Förster resonance energy transfer (smFRET) experiments (Fig. 1c), as well as high-field ${ }^{15} \mathrm{~N}$ spin relaxation rates (Fig. 1f-h) were measured in the current study.

First, we asked whether the motions sampled in the MD trajectory are in agreement with experimental ${ }^{1} \mathrm{H}$ relaxometry data. To this end, we calculated from the MD trajectory angular autocorrelation functions (ACFs) and spectral density functions for all $\mathrm{H} \alpha$, methylene and methyl protons. From the spectral density functions, individual ${ }^{1} \mathrm{H} R_{1}$ rates were predicted at different fields (SI, Methods). The calculated ACFs required a 4exponential decay function to be fitted (SI, Fig. S2a), with the longest correlation times showing a large variation (SI, Fig. S2). The average second correlation time was $1.6 \mathrm{~ns}$. The global $R_{1}$ dispersion profile was then simulated and compared with experiment (Fig. 1b). When the simulated profile was fitted to a single correlation time equation ( $\mathrm{SI}$, eq. S6a) ${ }^{[7]}$, it resulted in $\mathrm{Sc}^{2}$ $=0.10 \pm 0.01, \tau_{\mathrm{R}}=5.8 \pm 0.3 \mathrm{~ns}$ and $\alpha=5.0 \pm 0.5 \mathrm{~s}^{-1}$. These values are in good agreement with the parameters obtained from a fit of the experimental profile $\left(S_{c}{ }^{2}=0.08 \pm 0.01, \tau_{R}=6.3 \pm 0.4 \mathrm{~ns}\right.$ and $\alpha$ $=6.1 \pm 0.3 \mathrm{~s}^{-1}$ in water solution) ${ }^{[6]}$. The quality of the fit to the MD-predicted relaxometry profile improved when two correlation times were included (SI, eq. S6b). This is expected because a distribution of correlation times is at the origin of the simulated data (SI, Fig. S2b). Subsequently, the shorter correlation time ( $\left.\tau_{R 2}\right)$ was fixed to $1.6 \mathrm{~ns}$ (see above), because of its large covariance with the corresponding order parameter, $S_{c(2)}{ }^{2}$. The resulting best fit values for $\tau_{\mathrm{R} 1}, S_{\mathrm{C}(1)}{ }^{2}$ and $S_{\mathrm{C}(2)}{ }^{2}$ were $8.7 \pm 0.5 \mathrm{~ns}$, $0.044 \pm 0.004$ and $0.25 \pm 0.02$, respectively. When the experimental profile was similarly fitted, the best fit values for $\tau_{\mathrm{R} 1}$, $\left.S_{\mathrm{C}(1)}\right)^{2}$ and $S_{\mathrm{C}(2)}{ }^{2}$ were $8.6 \pm 1.6 \mathrm{~ns}, 0.05 \pm 0.02$ and $0.16 \pm 0.09$, respectively. The analysis shows that a state-of-the-art MD force field/water model is capable of capturing slow motions, which modulate ${ }^{1} \mathrm{H}-{ }^{1} \mathrm{H}$ dipolar interactions with a $\tau_{R}$ of $6-9 \mathrm{~ns}$.

smFRET and nsFCS have recently emerged as powerful techniques to study disordered proteins ${ }^{[8]}$. They rely on the distance-dependent coupling of the electronic transition dipoles of a donor and acceptor fluorophore attached to the polypeptide chain and enable access to longer timescales than ${ }^{1} \mathrm{H}$ relaxometry, typically in the range of tens to hundreds of nanoseconds for IDPs. To allow labeling of aS with Alexa $488 / 594$ as donor/acceptor, we prepared a double-cysteine variant of aS (S42C/T92C aS). In smFRET a mean transfer 
a Molecular Dynamics

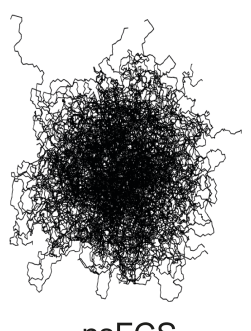

C
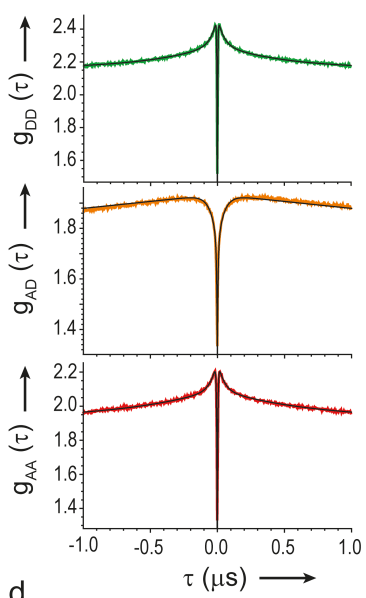

d

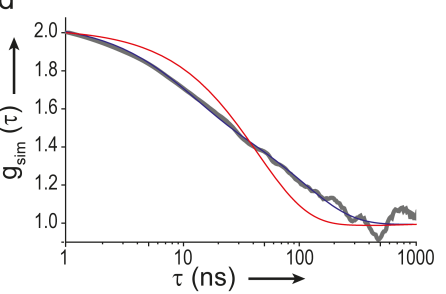

b proton relaxometry

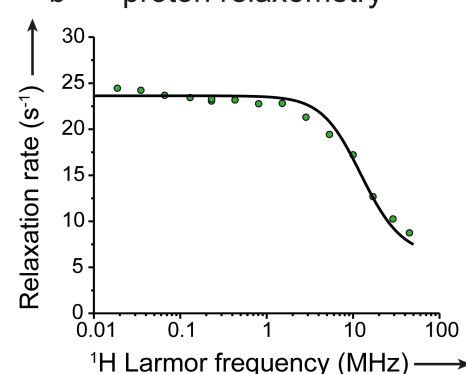

e ${ }^{15} \mathrm{~N}$ relaxation

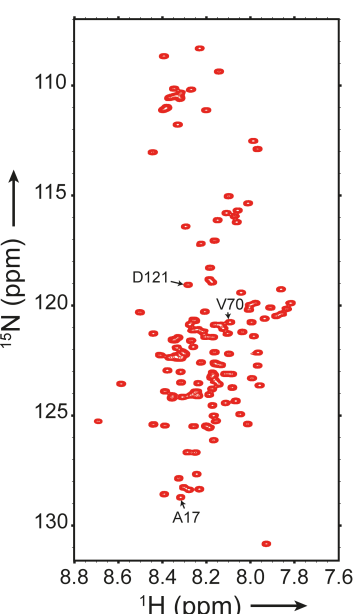

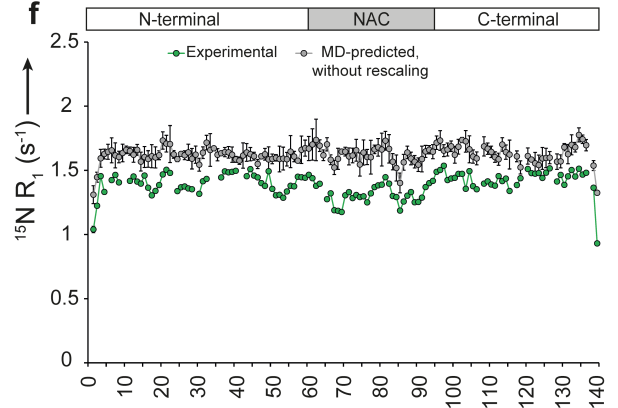

g

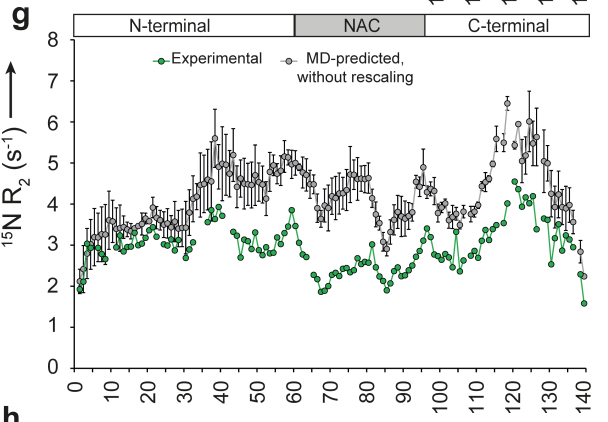

h

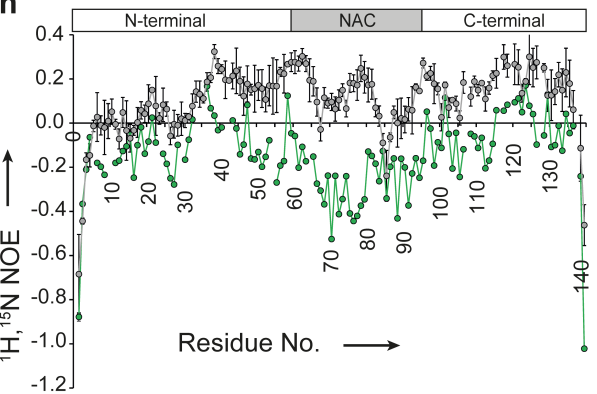

Figure 1. Integrative analysis of the local and global dynamics of aS. (a) Representative ensemble of aS from a $16-\mu s-l o n g$ MD simulation. (b) ${ }^{1} \mathrm{H}$ relaxometry measured in $\mathrm{D}_{2} \mathrm{O}$ (circles) superimposed on the MD simulated profile (solid line; rescaled to account for different viscosities of $\mathrm{H}_{2} \mathrm{O}$ and $\mathrm{D}_{2} \mathrm{O}$ ). (c,d) Comparison of experimental nsFCS (c) with the auto-correlation of the S42-T92 $\mathrm{C}^{\alpha}-\mathrm{C}^{\alpha}$ distance calculated from the MD trajectory (d). As compared to a single-exponential decay (red curve), the fit with a two-exponential decay function (purple curve) improved. Derived correlation times are similar to those obtained from the global fit of the experimental auto- and cross-correlations. (e) A typical ${ }^{15} \mathrm{~N},{ }^{1} \mathrm{H}$ correlation spectrum, with three residues from the $\mathrm{N}$-terminal, NAC and C-terminal regions (A17, V70 and D121) highlighted (see SI, Fig. S4). (f-h) ${ }^{15} \mathrm{~N} R_{1}$ and $R_{2}$ rates and hetNOEs, measured (green circles/lines) and calculated from the MD trajectory (orange triangles; at $600 \mathrm{MHz}$ ). Error bars for simulated $R_{1} / R_{2}$ represent the standard deviation between rates calculated from three MD sub-trajectories, each of $5 \mu \mathrm{s}$ duration. Error bars for experimental rates are smaller than the symbol size.

efficiency of 0.64 was obtained, corresponding to a root-meansquared (rms) inter-residue distance of $4.5 \pm 0.4 \mathrm{~nm}$, close to the rms distance of $4.4 \pm 0.2 \mathrm{~nm}$ calculated from the MD simulation (SI, Fig. S3 and Table S1). The observed fluorescence intensity correlation functions showed characteristic correlated and anticorrelated components in the sub-microsecond range in the auto-correlation and donor-acceptor cross-correlation functions, respectively (Fig. 1c). From a global analysis of all correlation functions obtained by nsFCS with a single-exponential decay, a reconfiguration time $\tau_{r}$ of $58 \pm 13 \mathrm{~ns}$ was determined. The $\tau_{r}$ value of aS is within the typical range obtained for other disordered proteins of similar length ${ }^{[\mathrm{bb}, 9]}$ and can be directly compared with the corresponding distance correlation time from the MD simulation of aS. Indeed, we obtained a mean relaxation time of $43 \mathrm{~ns}$ (Fig. 1d) from a single-exponential fit of the MD distance correlation, only slightly smaller than the experimental value. However, we also observed that the MD-based correlation function was better described by a double-exponential decay with two correlation times of $10 \pm 2 \mathrm{~ns}$ (amplitude $41 \pm 5 \%$ ) and $107 \pm 16$ ns $(59 \pm 5 \%)$. A similar analysis of the experimental correlations resulted in correlation times of $23 \pm 4 \mathrm{~ns}(66 \pm 2 \%)$ and $136 \pm 33 \mathrm{~ns}(34 \pm 2 \%)$. The weighted average of the two correlation times was $67 \pm 12$ ns from MD simulation and $61 \pm 14$ ns from nsFCS data. The analyses/measurements suggest that the MD trajectory of aS captures with good accuracy the motional timescales probed by ${ }^{1} \mathrm{H}$ relaxometry and nsFCS.

A particularly powerful technique for the study of proteins dynamics is high-field ${ }^{15} \mathrm{~N}$ spin relaxation, because it provides access to dynamics from tens of picoseconds to several nanoseconds with single-residue resolution ${ }^{[10]}$. We measured different ${ }^{15} \mathrm{~N}$ relaxation rates for aS at conditions identical to those used in the ${ }^{1} \mathrm{H}$ relaxometry experiments $(1 \mathrm{mM}$ aS, $\mathrm{pH} 5.0$, $25^{\circ} \mathrm{C}$; $\left.{ }^{[6]}\right)$. Consistent with previous reports ${ }^{[11]}$, the central NAC region (residues 60-95) exhibited transverse relaxation $\left(R_{2}\right)$ 
rates smaller than the average, while residues 115-130 in the Cterminus had larger $R_{2}$ rates (Fig. 1d-h; SI, Figs. S4,S5) [11a] Importantly, highly similar relaxation rates were obtained at lower aS concentration (150 $\mu \mathrm{M}$, SI, Fig. S6), excluding significant contributions from oligomerization. In addition, similar $R_{2}$ rates were derived from cross-correlated relaxation measurements (SI, Fig. S7), indicating that exchange processes do not contribute to the observed $R_{2}$ rates.

Several theoretical frameworks have been developed for the analysis of ${ }^{15} \mathrm{~N}$ relaxation data in IDPs ${ }^{[10 b, 12]}$. We first performed spectral density mapping ${ }^{[13]}$. The analysis showed that aS backbone dynamics cannot be described by a single correlation time, consistent with the disordered nature of aS (SI, Fig. S8). Next, we calculated angular ACFs for 134 individual backbone $\mathrm{N}-\mathrm{Hs}$ of aS from the MD trajectory (see SI, Methods, Fig. S9 and Table S2). On the basis of a three-exponential fit, average correlation times for the fast, intermediate and slow motions of $145 \pm 42$ ps, $1.53 \pm 0.43$ ns and $7.02 \pm 1.82$ ns were obtained. Corresponding order parameters were $0.26 \pm 0.05,0.49 \pm 0.04$ and $0.26 \pm 0.07$ for $S^{2}$ fast, $S^{2}$ int and $S^{2}$ slow, respectively (SI, Fig. S10).

Individual ACFs were also directly converted to spectral density functions through Fourier transformation, and residuespecific ${ }^{15} \mathrm{~N}$ relaxation rates were predicted and compared with experiment (Fig. 1f,g; SI, Fig. S5). Predicted ${ }^{15} \mathrm{~N} R_{2}$ and $C C R$, and to a lesser extent $R_{1}$ values, were larger than the experimental values, in agreement with an overestimation of $J(0)$ and $J\left(\omega_{N}\right)$ by $M D(S I$, Fig. S8). The comparison between experimental and predicted hetNOEs further indicated that the fast sub-nanosecond motions represented by $J\left(\omega_{H}\right)$ are underestimated by the MD trajectory (Fig. $1 \mathrm{~h}$ ). The origin of these discrepancies could be limitations in accurately capturing the timescales or amplitudes of motions in the MD trajectory.

With regard to potential temporal limitations, we found that a scaling factor of $\sim 0.55$ of the time axis of the MD trajectory would be required to match the experimental average ${ }^{15} \mathrm{~N}$ relaxation rates (SI, Fig. S11 and Table S3). However, this seems excluded by the good agreement between the correlation times obtained from experimental and MD-based ${ }^{1} \mathrm{H}$ relaxometry profiles and nsFCS data. We therefore used the ${ }^{15} \mathrm{~N}$ relaxation data to investigate potential restrictions of the backbone flexibility in MD. On the basis of $R_{1}$ and $R_{2}$ at $600 \mathrm{MHz}$, residuespecific scaling factors for $S^{2}$ fast, $S^{2}$ int and $S^{2}$ slow were determined (Fig. 2a, SI, Fig. S12a), while the scaling factor for the time axis was $1.05 \pm 0.05$ (SI, Fig. S12b), i.e. effectively no scaling for the time axis in agreement with the results comparing ${ }^{1} \mathrm{H}$ relaxometry and nsFCS with MD. Notably, a global rescaling factor for example for $S^{2}$ slow was not sufficient (SI, Fig. S11 and Table S3). This is expected because there are many modes of motion in protein backbone and side chains that overall affect $\mathrm{N}$ $\mathrm{H}$ reorientation in IDPs such that different residues in the sequence might experience different levels of reorientational dynamic limitations by current MD force fields.

Experimental ${ }^{15} \mathrm{~N}$ relaxation rates were best predicted from the MD simulation with scaling factors of $0.52 \pm 0.08$ for $S^{2}$ fast, $0.33 \pm 0.05$ for $S^{2}$ int and $0.15 \pm 0.06$ for $S^{2}$ slow. Cross-validation using experimental $C C R$ at $600 \mathrm{MHz}$ and $R_{2}$ at $800 \mathrm{MHz}$ demonstrated the reliability of the determined scaling factors (Fig. 2b and SI, Fig. S13). In addition, different regions of aS required different levels of scaling, with residues $60-90$ demanding the highest degree of $S^{2}$ slow scaling (SI, Fig. S14). The required order parameter scaling suggests that local flexibility is over-restricted in the MD simulation of aS.

Our study provides a detailed, residue-specific view of the backbone dynamics of the paradigmatic IDP aS. For most aS residues, fast motions reporting librational motions and local $\phi / \psi$ dihedral angle fluctuations decorrelate $\sim 52 \%$ of $\mathrm{N}-\mathrm{H}$ orientational memory, intermediate motions with correlation times of $\sim 1.6 \mathrm{~ns}$, reporting broader conformational sampling within and between Ramachandran substates, decorrelate $-33 \%$ of $\mathrm{N}-\mathrm{H}$ orientations, and $\sim 15 \%$ of correlation is removed by slow motions with correlation times of $\sim 6-13 \mathrm{~ns}$, representing segmental dynamics of the disordered aS chain (Fig. 3). In addition, we found that the NAC region possesses smaller amplitudes of slow motions $\left(S^{2}\right.$ slow $\left.=0.09 \pm 0.02\right)$ than the $\mathrm{N}$ - and $\mathrm{C}$-terminal residues. The conversion from an intrinsically disordered structure in solution to an ordered structure in as fibrils demands extensive conformational rearrangements, in which the high level of backbone dynamics in the NAC region, which forms the core of aS fibrils ${ }^{[14]}$, may play a supporting role. Another finding from our study is that five residues (Y39, V40, T44, T54 and V55) in the $\mathrm{N}$-terminal half of aS have very large $\tau_{\text {slow }}$ values, exceeding 10 ns (Fig. 3a). We note that Y39 plays an important role for aggregation of aS into amyloid fibrils ${ }^{[15]}$. Although we currently don't understand the detailed connection between backbone dynamics of aS and its pathological aggregation, the data suggest that local and global dynamics in the monomeric state of aS might be important for its pathogenic aggregation.

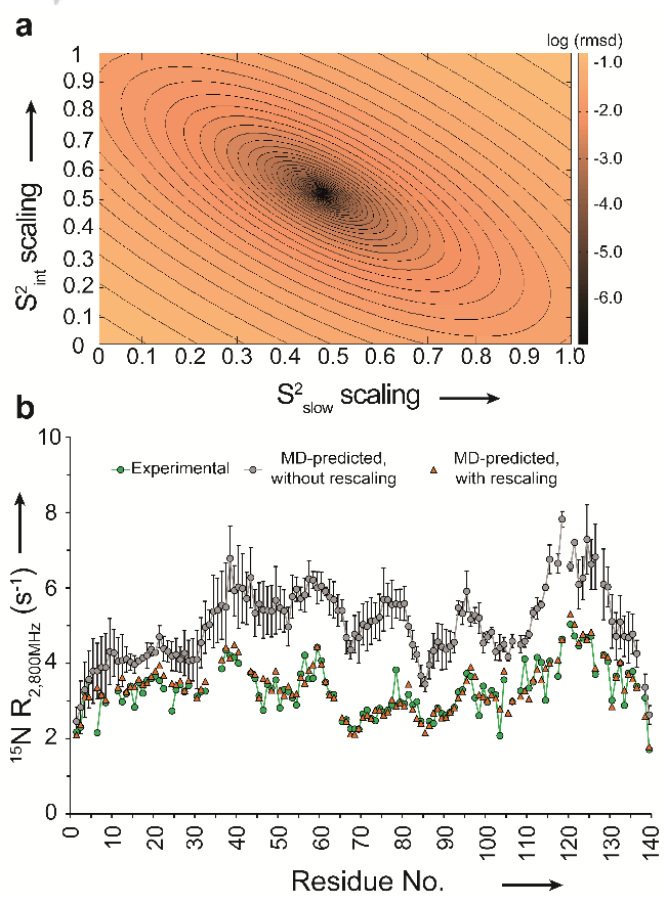

Figure 2. Order parameter optimization on the basis of ${ }^{15} \mathrm{~N} R_{1}$ and $R_{2}$ (at 600 $\mathrm{MHz}$ ) of aS. (a) Fit quality depends on rescaling of intermediate and slow squared order parameters $\left(S^{2}\right.$ int, $S^{2}$ slow $)$, shown here for $Y 39$. A minimum was found for $S^{2}$ int and $S^{2}$ slow scaling factors of $\sim 0.52$ and 0.48 , respectively. (b) Cross-validation by ${ }^{15} \mathrm{~N} R_{2}$ at $800 \mathrm{MHz}$. Simulated rates calculated on the basis of rescaled order parameters (red) match experimental rates (green). 
a

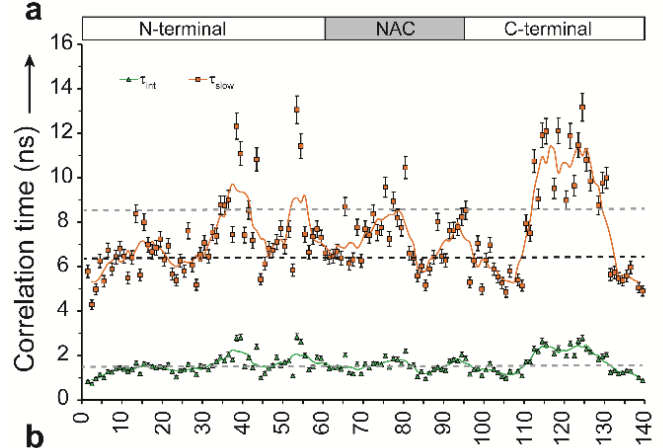

b

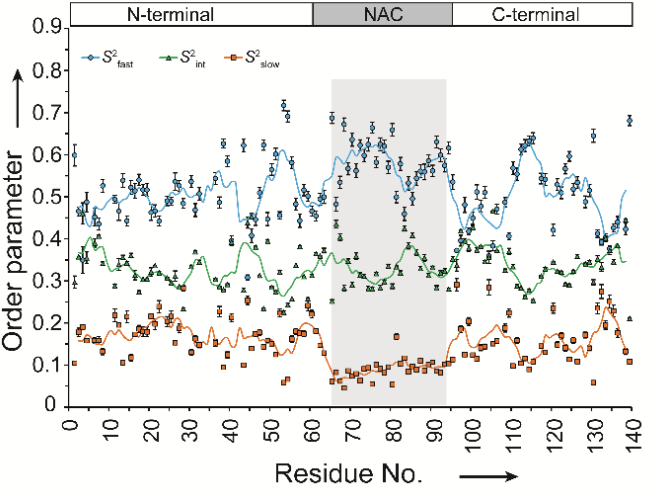

state of proteins paves the way for a high-resolution understanding of protein folding and misfolding.

\section{Acknowledgements}

This work was supported by the advanced grant 'LLPS-NMR' of the European Research Council (to M.Z.), the Fondazione Cassa di Risparmio di Firenze, MIUR PRIN 2012SK7ASN, European Commission (contracts Propag-ageing 634821 and iNEXT 653706), COST Action CA15209 (EURELAX), the EU ESFRI Instruct Core Centre CERM, the Swiss National Science Foundation (to B.S.), and the Deutsche Forschungsgemeinschaft (RE 3655/2-1, to N.R-G.). We thank D.E. Shaw Research for sharing their a-synuclein simulation data and Karin Giller for excellent technical help with protein preparation.

Keywords: intrinsically disordered protein $•$ protein dynamics $•$ NMR spectroscopy

[1] S. DeForte, V. N. Uversky, Molecules 2016, 21

[2] I. C. Felli, R. Pierattelli, Intrinsically Disordered Proteins Studied by NMR Spectroscopy, 1st ed., Springer, 2015.

[3] aN. Rezaei-Ghaleh, M. Blackledge, M. Zweckstetter, Chembiochem 2012, 13, 930-950; bM. R. Jensen, R. W. Ruigrok, M. Blackledge, Curr Opin Struct Biol 2013, 23, 426-435.

[4] aL. Salmon, G. Nodet, V. Ozenne, G. Yin, M. R. Jensen, M. Zweckstetter, M. Blackledge, J Am Chem Soc 2010, 132, 8407-8418; bJ. R. Allison, P. Varnai, C. M. Dobson, M. Vendruscolo, J Am Chem Soc 2009, 131, 18314-18326; cO. Ullman, C. K. Fisher, C. M. Stultz, J Am Chem Soc 2011, 133, 1953619546; dT. Hosek, E. O. Calcada, M. O. Nogueira, M. Salvi, T. D. Pagani, I. C. Felli, R. Pierattelli, Chemistry 2016, 22, 13010-13013.

[5] S. Piana, A. G. Donchev, P. Robustelli, D. E. Shaw, J Phys Chem B 2015, 119, 5113-5123.

[6] G. Parigi, N. Rezaei-Ghaleh, A. Giachetti, S. Becker, C. Fernandez, M. Blackledge, C. Griesinger, M. Zweckstetter, C. Luchinat, J Am Chem Soc 2014, 136, 16201-16209.

[7] C. Luchinat, G. Parigi, J Am Chem Soc 2007, 129, 1055-1064.

[8] aH. S. Chung, K. McHale, J. M. Louis, W. A. Eaton, Science 2012, 335, 981984; bD. Nettels, I. V. Gopich, A. Hoffmann, B. Schuler, Proc Natl Acad Sci U $S$ A 2007, 104, 2655-2660.

[9] aA. Soranno, B. Buchli, D. Nettels, R. R. Cheng, S. Muller-Spath, S. H. Pfeil, A. Hoffmann, E. A. Lipman, D. E. Makarov, B. Schuler, Proc Natl Acad Sci U S A 2012, 109, 17800-17806; bM. Aznauryan, L. Delgado, A. Soranno, D. Nettels, J. R. Huang, A. M. Labhardt, S. Grzesiek, B. Schuler, Proc Natl Acad Sci U S A 2016, 113, E5389-5398; cB. Schuler, H. Hofmann, A. Soranno, D. Nettels, Annu Rev Biophys 2016, 45, 207-231; dA. Soranno, A. Holla, F. Dingfelder, D. Nettels, D. E. Makarov, B. Schuler, Proc Natl Acad Sci U S A 2017, 114, E1833-E1839.

[10] aN. Salvi, A. Abyzov, M. Blackledge, Prog Nucl Magn Reson Spectrosc 2017, 102-103, 43-60; bM. L. Gill, R. A. Byrd, A. G. Palmer, III, Phys Chem Chem Phys 2016, 18, 5839-5849.

[11] aR. Bussell, Jr., D. Eliezer, J Biol Chem 2001, 276, 45996-46003; bS. McClendon, C. C. Rospigliosi, D. Eliezer, Protein Sci 2009, 18, 1531-1540; cT. S. Ulmer, A. Bax, J Biol Chem 2005, 280, 43179-43187.

[12] aN. A. Farrow, O. Zhang, J. D. Forman-Kay, L. E. Kay, Biochemistry 1997 36, 2390-2402; bP. Kaderavek, V. Zapletal, A. Rabatinova, L. Krasny, V. Sklenar, L. Zidek, J Biomol NMR 2014, 58, 193-207; cM. Buck, H. Schwalbe, C. M. Dobson, J Mol Biol 1996, 257, 669-683; dK. Modig, F. M. Poulsen, J Biomol NMR 2008, 42, 163-177; eS. N. Khan, C. Charlier, R. Augustyniak, N. 
Salvi, V. Dejean, G. Bodenhausen, O. Lequin, P. Pelupessy, F. Ferrage, Biophys J 2015, 109, 988-999; fJ. J. Prompers, R. Bruschweiler, J Am Chem Soc 2002, 124, 4522-4534

[13] N. A. Farrow, O. Zhang, J. D. Forman-Kay, L. E. Kay, Biochemistry 1995, 34, 868-878.

[14] M. D. Tuttle, G. Comellas, A. J. Nieuwkoop, D. J. Covell, D. A. Berthold, K. D. Kloepper, J. M. Courtney, J. K. Kim, A. M. Barclay, A. Kendall, W. Wan, G. Stubbs, C. D. Schwieters, V. M. Lee, J. M. George, C. M. Rienstra, Nat Struct Mol Biol 2016, 23, 409-415.

[15] R. M. Rasia, C. W. Bertoncini, D. Marsh, W. Hoyer, D. Cherny, M. Zweckstetter, C. Griesinger, T. M. Jovin, C. O. Fernandez, Proc Natl Acad Sci U S A 2005, 102, 4294-4299.

[16] G. Fusco, A. De Simone, T. Gopinath, V. Vostrikov, M. Vendruscolo, C. M. Dobson, G. Veglia, Nat Commun 2014, 5, 3827

[17] B. A. Shoemaker, J. J. Portman, P. G. Wolynes, Proc Natl Acad Sci U S A 2000, 97, 8868-8873.

[18] Y. Gu, D. W. Li, R. Bruschweiler, J Chem Theory Comput 2014, 10, 25992607.

[19] N. Salvi, A. Abyzov, M. Blackledge, J Phys Chem Lett 2016, 2483-2489. 
Capturing local and global dynamics from one to hundreds of nanoseconds in the intrinsically disordered protein $\alpha$-synuclein: the combination of three experimental techniques, which are sensitive to motions on different length and timescales, with long molecular dynamics simulations is a powerful strategy to determine residue-specific protein dynamics in IDPs at different time and length scales.

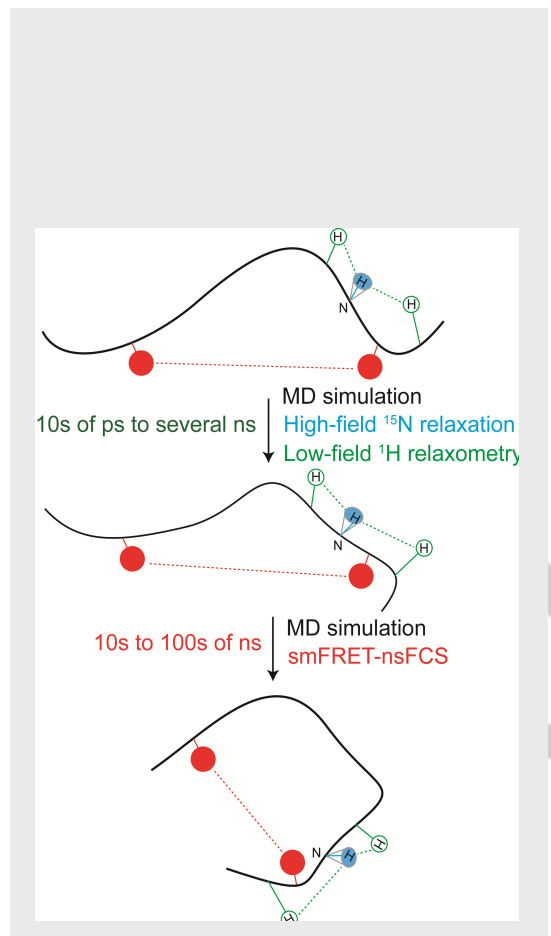

Nasrollah Rezaei-Ghaleh, Giacomo Parigi, Andrea Soranno, Andrea Holla, Stefan Becker, Benjamin Schuler, Claudio Luchinat*, Markus Zweckstetter ${ }^{*}$

Page No. - Page No.

Local and Global Dynamics in Intrinsically Disordered synuclein 